\title{
Autofluorescencia retiniana en la evaluación de la respuesta al tratamiento del edema macular diabético
}

\author{
Sergio E. Hernández-Da Mota, ${ }^{1}$ Francisco Béjar-Cornejo, ${ }^{1}$ Virgilio Lima-Gómez, ${ }^{2}$ \\ Ernesto Rodríguez-Ayala ${ }^{3}$ \\ ${ }^{1}$ Clínica David, Servicio de Retina, Morelia, Michoacán; ${ }^{2}$ Universidad Michoacana de "San Nicolás de Hidalgo", Facultad de Medicina "Dr. Ignacio \\ Chávez", Morelia, Michoacán; ${ }^{3}$ Hospital Juárez de México, Servicio de Oftalmología, Ciudad de México. México
}

\section{Resumen}

Introducción: La autofluorescencia retiniana $(A F)$ ha mostrado sensibilidad en la detección del edema macular. Objetivos: Evaluar índices formados con la AF y variables anatomofuncionales retinianas en pacientes con edema macular diabético (EMD) tratados con ziv-aflibercept (ziv-AFL). Métodos: Fueron incluidos 29 ojos de 15 pacientes con EMD que recibieron inyecciones intravítreas de ziv-AFL. Se evaluó agudeza visual mejor corregida (AVMC), sensibilidad al contraste (SC), tomografía de coherencia óptica (TCO) y AF, antes del tratamiento, así como al primer y segundo mes de iniciado este. Las variables de la TCO fueron grosor foveal central (GFC), volumen macular (VM) y grosor promedio macular (GPM). Se obtuvieron los valores basales de AF/AVMC, AF/SC, AF/GFC, AF/VM y AF/GPM. Se realizó análisis con el coeficiente de correlación de rangos de Spearman y análisis de regresión lineal. Resultados: Hubo una correlación significativa entre el índice AF/AVMC basal y la AVMC en el segundo mes $\left(r_{s}=-0.78, p=0.000\right)$, entre el índice AF/SC basal y la AVMC en el segundo mes $\left(r_{s}=-0.68, p=0.0009\right)$ $y$ entre AF/SC basal y el VM en el primer mes de seguimiento $\left(r_{s}=0.64, p=0.002\right)$. Conclusiones: En el EMD, los índices compuestos con AF basales predicen variables como AVMC en el seguimiento de pacientes que reciben ziv-AFL.

PALABRAS CLAVE: Edema macular diabético. Autofluorescencia retiniana. Tomografia de coherencia óptica de dominio espectral. Ziv-aflibercept. Terapia anti-VEGF intravítrea.

\section{Fundus autofluorescence in the evaluation of diabetic macular edema treatment response}

\begin{abstract}
Introduction: Fundus autofluorescence (FAF) has shown sensitivity in the detection of macular edema. Objectives: To evaluate indices formed with $F A F$ and retinal anatomical-functional variables in patients with diabetic macular edema (DME) treated with Ziv-aflibercept (Ziv-AFL). Methods: Twenty-nine eyes of 15 DME patients who received Ziv-AFL intravitreal injections were included. Best-corrected visual acuity (BCVA), contrast sensitivity (CS), optical coherence tomography (OCT), and FAF were evaluated before treatment and at one and two months. OCT variables were central subfield thickness (CST), macular volume (MV), and macular cube average thickness (MCAT). FAF/BCVA, FAF/CS, FAF/CST, FAF/MV and AF/MCAT indices baseline values were obtained. Analysis was performed with Spearman's rank correlation coefficient and linear regression analysis. Results: There was a significant correlation between baseline FAF/BCVA index and BCVA at second month $\left(r_{s}=-0.78, p=0.000\right)$, between baseline FAF/CS index and BCVA at second month $\left(r_{s}=-0.68, p=0.0009\right)$, and between baseline FAF/CS index and MV at first month of follow-up $\left(r_{s}=0.64, p=0.002\right)$. Conclusions: In DME, composite indices with baseline FAF predict variables such as BCVA in the follow-up of patients receiving ziv-AFL.
\end{abstract}

KEY WORDS: Diabetic macular edema. Fundus autofluorescence. SD-OCT. Ziv-aflibercept. Intravitreal anti-VEGF therapy.

Correspondencia:

*Sergio E. Hernández-Da Mota

E-mail: tolodamota@yahoo.com.mx
Fecha de recepción: 21-11-2020

Fecha de aceptación: 08-12-2020

DOI: $10.24875 / G M M .20000844$
Gac Med Mex. 2021;157:411-417

Disponible en PubMed

www.gacetamedicademexico.com

0016-3813/@ 2021 Academia Nacional de Medicina de México, A.C. Publicado por Permanyer. Este es un artículo open access bajo la licencia CC BY-NC-ND (http://creativecommons.org/licenses/by-nc-nd/4.0/). 


\section{Introducción}

El edema macular diabético (EMD) es la principal causa de baja visual en los pacientes diabéticos. ${ }^{1,2}$ Afecta aproximadamente a $30 \%$ de los pacientes con más de 20 años de padecer diabetes mellitus. ${ }^{3}$ La biomicroscopia con lámpara de hendidura, la angiografía de fluoresceína y la tomografía de coherencia óptica (TCO) son métodos que han demostrado utilidad en el diagnóstico y seguimiento de los pacientes con EMD. ${ }^{4,5}$

La autofluorescencia retiniana (AF) es una modalidad diagnóstica empleada para la evaluación integral de varias patologías retinianas, ${ }^{6}$ la cual ha mostrado cierta sensibilidad en la detección del edema macular quístico, principalmente con una longitud de onda corta. $^{7}$

Por lo tanto, la AF puede representar una técnica no invasiva para la obtención de imágenes y la evaluación de la respuesta a la terapia antiangiogénica intravítrea en el EMD.

Las imágenes obtenidas con sistemas que emplean una longitud de onda más larga basados en cámara de flash se correlacionan con parámetros de TCO en la detección del EMD, ${ }^{8,9}$ pero tienen una sensibilidad menor en comparación con las de longitud de onda más corta. ${ }^{7}$

Los índices formados por el cociente del grado de AF y variables como la agudeza visual mejor corregida (AVMC) o el volumen macular (VM) pudieran ser de utilidad como características o biomarcadores basales que ayuden a predecir la respuesta al tratamiento con terapia antiangiogénica en pacientes con EMD, en comparación con las variables no combinadas con la AF, como la AVMC o la sensibilidad al contraste (SC) basales.

El objetivo del presente estudio fue evaluar las correlaciones entre los índices compuestos con AF y otras variables anatomofuncionales en pacientes con EMD bajo tratamiento con inyecciones intravítreas de ziv-aflibercept (ziv-AFL).

\section{Métodos}

Subanálisis retrospectivo de una serie de casos previamente publicada por nuestro grupo. ${ }^{8,9} \mathrm{Se}$ incluyeron 29 ojos de 15 pacientes reclutados en el Servicio de Retina de la Clínica David, Unidad Oftalmológica, en la Ciudad de Morelia, Michoacán, México.
Fueron incluidos pacientes que recibieron inyecciones intravítreas de ziv-AFL para el tratamiento de EMD (definido como un engrosamiento $>260 \mu \mathrm{m}$ del subcampo foveal central). Se excluyeron los pacientes con cualquier tratamiento láser en la mácula (dentro de un diámetro de $1500 \mu \mathrm{m}$ alrededor del centro foveal) o cirugía intraocular realizada seis meses antes del inicio del tratamiento con inyecciones intravítreas de ziv-AFL.

A todos los pacientes se les realizó un examen oftalmológico completo que incluyó toma de AVMC, examen del segmento anterior, oftalmoscopia indirecta y biomicroscopia con lente de 78D. Se tomaron imágenes de AF, TCO dominio espectral y fotografía clínica del polo posterior.

\section{Procedimientos realizados durante el estudio}

- Todos los pacientes recibieron tres inyecciones intravítreas de ziv-AFL bajo condiciones estériles el mismo día de la evaluación basal, así como al primer y segundo mes de seguimiento. Se empleó un método estandarizado previamente descrito ${ }^{8-11}$ para la preparación del sitio de la inyección y su aplicación.

- La AVMC lejana para cada ojo fue tomada con el protocolo estandarizado del Early Treatment Diabetic Retinopathy Study (ETDRS) a una distancia de $4 \mathrm{~m}$, con una cartilla para visión lejana modificada del ETDRS (Precision Vision, La Salle, Illinois, Estados Unidos). La SC fue obtenida con la cartilla Hamilton-Veale para la evaluación de SC (Hamilton Veale, Canterbury, Nueva Zelanda). Se realizaron tomas basales, así como al primer y segundo mes, tanto de AVMC como de SC.

- Autofluorescencia retiniana. Las imágenes de AF fueron obtenidas con una cámara de fondo (VISUCAM $^{\text {बNM/FA }}$, Carl Zeiss Meditec Inc., Oberkochen, Alemania), con una longitud de onda de excitación de 510 a $580 \mathrm{~nm}$ y una detección de la luz emitida arriba de los $640 \mathrm{~nm}$. Se tomaron imágenes de AF, basales, al primer y segundo mes.

- Las imágenes se estadificaron de acuerdo con diferentes patrones de AF foveal, a partir de una clasificación modificada basada en la descrita por Vujosevic et al. ${ }^{10}$ y Hernández-Da Mota et al..$^{8-10}$ Los grados de la clasificación fueron: grado 1 o AF disminuida (AFd); grado 2 o normal (AFn); grado 3 o AF aumentada unifocal (AFa unifocal); grado 4 o $\mathrm{AF}$ aumentada multifocal 
(AFa multifocal) y grado 5 o AF aumentada multifocal confluente o "en placa" (AFa en placa). La estadificación de las imágenes de AF fue realizada en forma independiente por dos evaluadores expertos en forma ciega.

- TCO. Las imágenes fueron obtenidas con el equipo modelo Cirrus ${ }^{\mathrm{TM}}$ HD-OCT 5000 (Carl Zeiss Meditec Inc., Oberkochen, Germany), en forma similar a las otras variables (se realizaron tomas basales, al primer y segundo mes).

El protocolo de escaneo fue el de grosor macular calculado con base en el programa de cubo macular $512 \times 128$. Para este estudio se obtuvo el valor del grosor del subcampo foveal central (GFC) en micras $(\mu \mathrm{m})$. También fueron registrados los valores del VM en $\mathrm{mm}^{3}$ y el grosor promedio macular (GPM) en $\mu \mathrm{m}$.

\section{Análisis estadístico}

Todos los datos fueron registrados y analizados usando el programa GraphPad Prism versión 8 (GraphPad Software Inc., San Diego, California). De todos los valores basales se obtuvieron promedios y su respectiva desviación estándar.

Los valores del grado de AF se convirtieron a su valor logarítmico correspondiente (grado $1=0$, grado $2=0.3$, grado $3=0.47$, grado $4=0.6$, grado $5=0.69$ ). ${ }^{9}$ Se obtuvieron índices basales con la división del valor logarítmico de la AF basal entre cada una de las otras variables basales (AVMC medida en número de letras, SC medida en número de pares de letras, GFC medida en $\mu \mathrm{m}$, VM medido en $\mathrm{mm}^{3}$ y GPM medido en $\mu \mathrm{m}$ ).

En el análisis de correlación se obtuvieron los coeficientes de correlación de rangos de Spearman, a partir de los índices basales obtenidos y las diferentes variables anatomofuncionales durante el seguimiento (primer y segundo mes).

Igualmente, se realizaron correlaciones entre los valores de las variables basales (AVMC, SC, GFC) y los valores de las variables (AVMC, VM y SC) obtenidos durante el seguimiento. Se aplicó análisis de regresión lineal y se evaluó la variabilidad interobservador (coeficiente kappa). Se empleó prueba Z para la comparación entre los diferentes coeficientes de correlación obtenidos.

\section{Resultados}

Veintinueve ojos de 15 pacientes fueron incluidos en esta serie de casos. El rango de edad de los pacientes fue de 53 a 74 años.
Tabla 1. Características basales de los pacientes con EMD incluidos en el estudio

Edad en años

Promedio $\pm \mathrm{DE}$

Rango

$61.8 \pm 6.2$

$53-74$

AVMC, número de letras en la cartilla

$32.3 \pm 16.3$ ETDRS

SC, número de pares de letras

$6.8 \pm 3.7$

Grosor foveal central por TCO $(\mu \mathrm{m})$

$390 \pm 118.8$

Volumen macular por TCO $\left(\mathrm{mm}^{3}\right)$

$11.2 \pm 3.2$

Grosor promedio macular por TCO $(\mu \mathrm{m})$

$383.8 \pm 95.7$

\begin{tabular}{|c|c|c|}
\hline & $\mathrm{n}$ & $\%$ \\
\hline \multicolumn{3}{|l|}{ Sexo } \\
\hline Masculino & 10 & 75 \\
\hline Femenino & 5 & 25 \\
\hline \multicolumn{3}{|l|}{ Patrón de AF } \\
\hline Grado 1 (disminuido) & 5 & 17.2 \\
\hline Grado 2 (normal) & 11 & 37.9 \\
\hline Grado 3 (aumentado unifocal) & 4 & 13.7 \\
\hline Grado 4 (aumentado multifocal) & 6 & 20.6 \\
\hline $\begin{array}{l}\text { Grado } 5 \text { (aumentado multifocal confluente o } \\
\text { "en placa") }\end{array}$ & 3 & 10.3 \\
\hline \multicolumn{3}{|l|}{ Patrones de edema por TCO } \\
\hline Quístico & 7 & 22.9 \\
\hline No quístico (espongiforme) & 17 & 60 \\
\hline Desprendimiento seroso de neuroretina & 5 & 17. \\
\hline
\end{tabular}
subfoveal

AVMC = agudeza visual mejor corregida, $\mathrm{SC}=$ sensibilidad al contraste $\mathrm{TCO}=$ tomografía de coherencia óptica, $\mathrm{AF}=$ autofluorescencia retiniana

AVMC, SC, GFC, VM y GPM, los patrones de AF y los patrones por TCO de edema macular basales de los pacientes incluidos en el estudio se muestran en la Tabla 1.

La variabilidad interobservador entre los dos evaluadores para estadificar la AF fue evaluada con el coeficiente kappa $(0.806, p=0.000)$.

Hubo correlaciones estadísticamente significativas entre los índices basales y algunas variables anatomofuncionales durante el seguimiento de los pacientes (Figuras 1 a 3). Las correlaciones más fuertes fueron entre el índice AF/AVMC basal y la AVMC en el segundo mes $\left(r_{s}=-0.78, p=0.0003\right.$, Figura 1), entre el índice AF/SC basal y el VM en el primer mes $\left(r_{s}=0.64, p=0.001\right.$, Figura 2) y entre el índice AF/ GFC basal y la SC en el primer mes $\left(r_{s}=0.44\right.$, $p=0.02$, Figura 3).

La correlación entre los índices AF/AVMC y AF/SC basales con el VM en el primer mes de seguimiento 
A

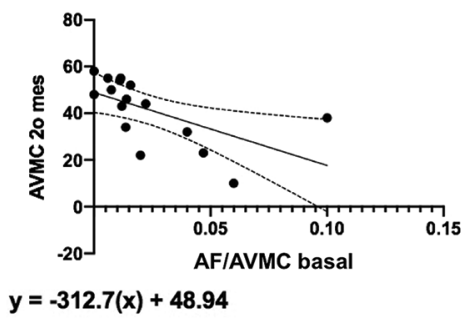

B

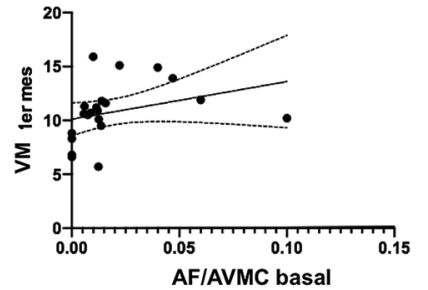

$y=34.89(x)+10.11$
C $r_{s}=0.8, p=0.0003,($ IC $950.47-0.93)$

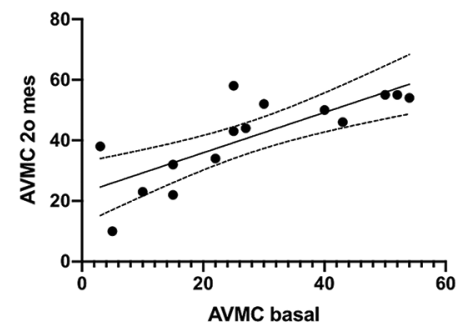

$y=0.6648(x)+22.63$

D

$r_{s}=-0.1, p=0.32,($ IC $95-0.54-0.37)$

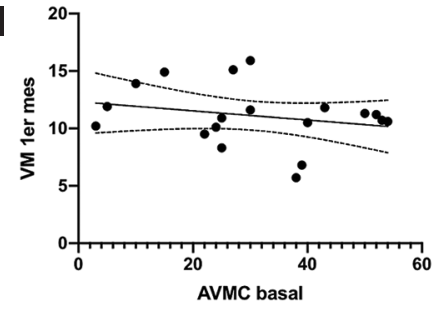

$y=-0.03990(x)+12.33$

Figura 1. A) Correlación entre índice AF/AVMC basal y AVMC en el segundo mes de seguimiento. B) Correlación entre el índice AF/AVMC basal y VM en el mes de seguimiento. C) Correlación entre AVMC basal y AVMC al segundo mes de seguimiento. D) Correlación entre la AVMC basal y VM en el primer mes.

A

$r_{s}=0.64, p=0.001($ IC $950.27-0.85)$

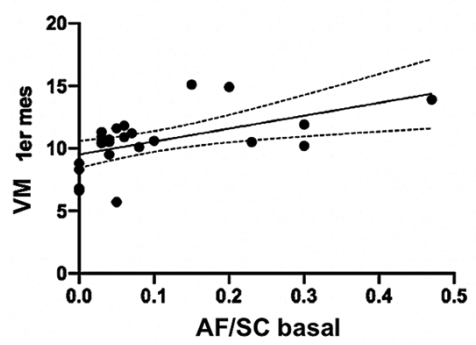

$y=10.33(x)+9.518$

B

$r_{s}=-0.61, p=0.0089,($ IC $95-0.86--0.13)$

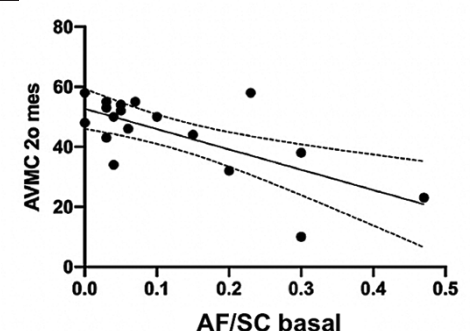

$y=-67.72(x)+52.7$
C

$r_{s}=-0.2, p=0.17,($ IC $95-0.63-0.27)$

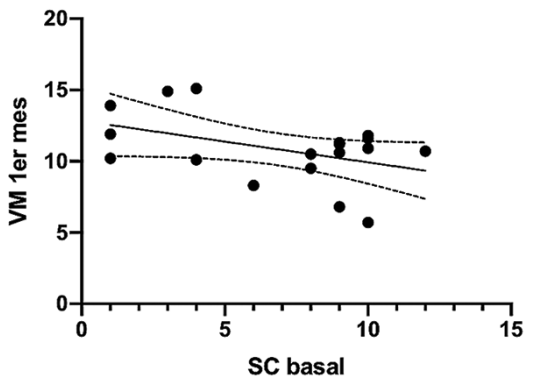

$y=-0.2910(x)+12.84$

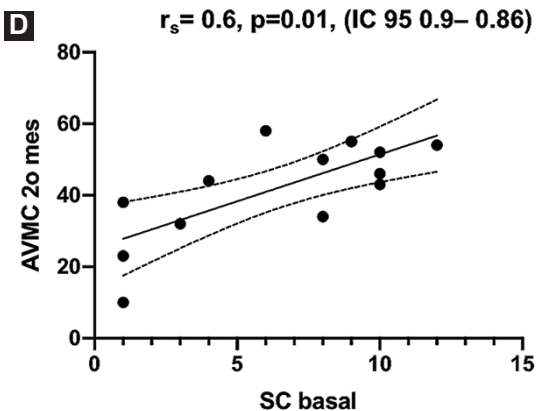

$y=2.624(x)+25.18$

Figura 2. A) Correlación entre índice AF/SC basal y VM en el primer mes de seguimiento. B) Correlación entre el índice AF/SC basal y VM en el primer mes de seguimiento. C) Correlación entre SC basal y VM en el primer mes de seguimiento. D) Correlación entre SC basal y AVMC en el segundo mes. 
A

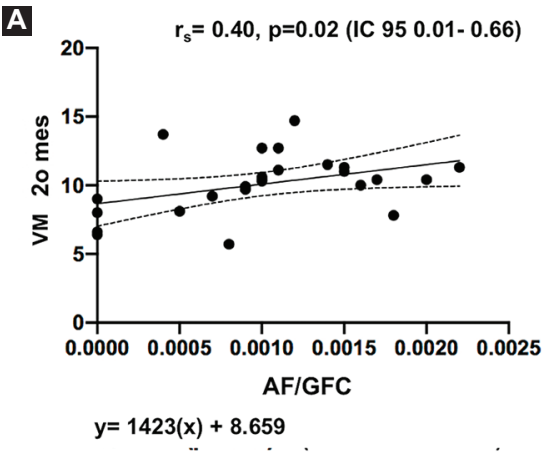

B

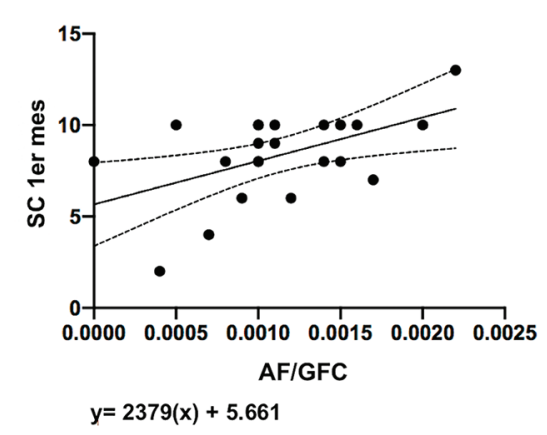

C

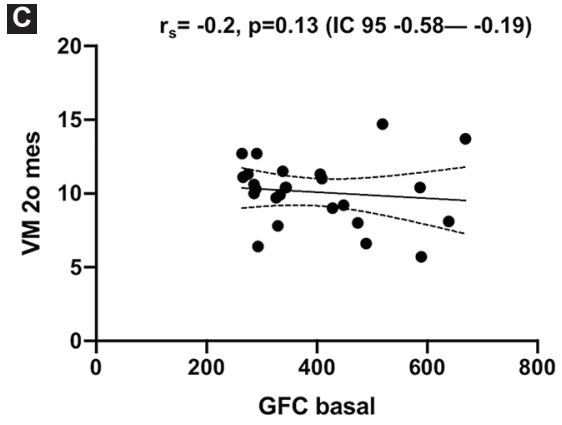

$y=-0.002085(x)+10.93$

D

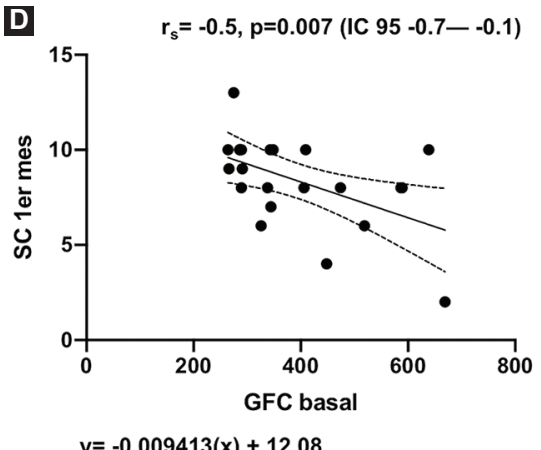

Figura 3. A) Correlación entre índice AF/GFC basal y VM en el segundo mes de seguimiento. B) Correlación entre índice AF/GFC basal y SC en el primer mes de seguimiento. C) Correlación entre GFC basal y VM en el segundo mes de seguimiento. D) Correlación entre GFC basal y SC en el primer mes.

fue mayor que la observada entre AVMC y SC basales y el VM en el primer mes de seguimiento (Figuras 1 y 2). En la Tabla 2 se muestran las comparaciones entre los diferentes coeficientes de correlación.

La comparación entre la correlación AF/AVMC basal y el VM en el primer mes $\left(r_{s}=0.56\right)$, con la correlación AVMC basal y el VM en el primer mes $\left(r_{s}=-0.1\right)$ fue la que mostró significación estadística $(p=0.000$, Tabla 2).

\section{Discusión}

La AF ha sido descrita como un método útil en la detección y seguimiento de enfermedades retinianas. ${ }^{6,8-12}$ No obstante, pocos estudios han abordado el papel de la AF en la evaluación de pacientes con EMD. ${ }^{13-17}$

Calvo-Maroto et al..$^{14}$ y Shen et al..$^{15}$ describieron los hallazgos y la clasificación de la AF en el EMD.

En el presente estudio se emplearon índices que toman en cuenta a la AF y se correlacionaron con variables anatomofuncionales, como algunas variables obtenidas en la TCO, para determinar la predicción de los resultados del tratamiento con ziv-AFL en
Tabla 2. Comparaciones entre las correlaciones

\begin{tabular}{|c|c|c|c|c|}
\hline Correlación & $r_{s}$ & Correlación & $r_{s}$ & $p$ \\
\hline $\begin{array}{l}\text { AF/AVMC basal-AVMC } \\
\text { segundo mes }\end{array}$ & -0.78 & $\begin{array}{c}\text { AVMC } \\
\text { basal-AVMC } \\
\text { segundo mes }\end{array}$ & 0.8 & 0.40 \\
\hline $\begin{array}{l}\text { AF/AVMC basal-VM } \\
\text { primer mes }\end{array}$ & 0.56 & $\begin{array}{l}\text { AVMC basal-VM } \\
\text { primer mes }\end{array}$ & -0.1 & 0.000 \\
\hline $\begin{array}{l}\text { AF/SC basal-VM } \\
\text { primer mes }\end{array}$ & 0.64 & $\begin{array}{l}\text { SC basal-VM } \\
\text { primer mes }\end{array}$ & -0.2 & 0.06 \\
\hline $\begin{array}{l}\text { AF/SC basal-AVMC } \\
\text { segundo mes }\end{array}$ & -0.61 & $\begin{array}{l}\text { SC basal-AVMC } \\
\text { segundo mes }\end{array}$ & 0.6 & 0.48 \\
\hline $\begin{array}{l}\text { AF/GFC basal-VM } \\
\text { segundo mes }\end{array}$ & 0.40 & $\begin{array}{l}\text { GFC basal-VM } \\
\text { segundo mes }\end{array}$ & -0.2 & 0.16 \\
\hline $\begin{array}{l}\text { AF/GFC basal-SC } \\
\text { primer mes }\end{array}$ & 0.44 & $\begin{array}{l}\text { GFC basal-SC } \\
\text { primer mes }\end{array}$ & -0.5 & 0.37 \\
\hline
\end{tabular}

$\mathrm{AF}=$ autofluorescencia retiniana, $\mathrm{AVMC}=$ agudeza visual mejor corregida,

$\mathrm{SC}=$ sensibilidad al contraste, $\mathrm{GFC}=$ grosor foveal central, $\mathrm{VM}=$ volumen macular .

pacientes con EMD. De la misma forma, se realizaron comparaciones entre las diferentes correlaciones.

En un estudio previo ${ }^{8}$ se demostró que la AF basal en el EMD obtenido se correlacionó en forma positiva con cambios estructurales de TCO. Esta correlación pudo predecir cambios en el grosor macular en el 
corto plazo en pacientes bajo tratamiento con terapia anti-VEGF; sin embargo, la correlación no fue tan fuerte como la descrita en otras publicaciones. ${ }^{17}$

Frampton et al. ${ }^{7}$ mostraron que una longitud de onda mayor en la adquisición de imágenes de AF puede traducirse en una menor sensibilidad para detectar alteraciones, lo cual, a su vez, pudiera reducir la probabilidad de detectar correlaciones significativas con otras variables. Para compensar esta limitación con los sistemas de cámara de flash, en el presente estudio se emplearon índices en los cuales se combinó el grado de AF con otras variables.

De acuerdo con nuestros resultados, los índices AF/SC y AF/AVMC basales tuvieron una correlación fuerte negativa con la AVMC en el seguimiento de los pacientes incluidos $\left(r_{s}\right.$ menor a -0.6). Se evidenció una correlación fuerte positiva entre el índice AF/SC basal y el VM en el primer mes de seguimiento $\left(r_{s}=0.64\right)$. De esta manera, los índices AF/AVMC y AF/SC basales podrían ayudar a predecir de forma más precisa la AVMC en el corto plazo (primer y segundo mes). Igualmente, el índice AF/SC pudiera predecir al VM en los pacientes bajo tratamiento con ziv-AFL para el EMD.

En un estudio previamente publicado por nuestro grupo, ${ }^{9}$ sugerimos que puede existir un beneficio potencial en aplicar algunos de los índices descritos como biomarcadores predictivos en pacientes con EMD.

El índice AF/AVMC mostró una correlación significativa con la AVMC a los dos meses $\left(r_{s}=-0.78\right.$, $p=0.0003)$. Esta correlación indica que el grado de AF basal puede ser directamente proporcional a la pérdida de AVMC. Por el contrario, la AVMC basal es inversamente proporcional a la AVMC presente a los dos meses de tratamiento con ziv-AFL. ${ }^{9}$

La comparación entre la correlación de AF/AVMC basal y VM en el primer mes $\left(r_{s}=0.56\right)$, con la correlación AVMC basal y el VM en el primer mes $\left(r_{s}=-0.1\right)$ fue la que mostró significación estadística. Lo anterior sugiere que el índice formado por la AF y la AVMC basales sería un mejor biomarcador basal para predecir la respuesta en disminución de VM al tratamiento con ziv-AFL que la AVMC basal.

Pece et al..$^{18}$ realizaron correlaciones cualitativas de imágenes de AF en edema macular diabético quístico (EMQ), con hallazgos de AF y de TCO. Concluyeron que con el sistema de obtención de imágenes de láser de barrido confocal que emplea una longitud de onda excitatoria corta $(488 \mathrm{~nm})$, se pueden visualizar espacios multilobulares con autofluorescencia aumentada en pacientes con EMQ.

Reznicek et al. ${ }^{12}$ evaluaron la AF de luz verde de igual forma en el EMD. Sus resultados indicaron que no hubo correlaciones significativas entre la intensidad de la AF y el GFC, la integridad de la capa elipsoide y el grosor de la capa del EPR-elipsoide en los pacientes diabéticos con y sin EMD. Por el contrario, en nuestros resultados, con una longitud de onda excitatoria más larga, se mostraron correlaciones estadísticamente significativas con el uso de los índices compuestos con el grado de AF.

Las correlaciones en nuestro estudio son consistentes con lo reportado por Chung et al. ${ }^{17}$ Las mediciones de AF se correlacionaron bien con variables cuantitativas $(r=0.7)$. Determinaron, por otro lado, que las asociaciones podían predecir la restauración de la integridad de la capa elipsoide con la subsecuente mejoría en la capacidad visual.

Por otra parte, Vujosevic et al..$^{11}$ determinaron que la AF se correlacionaba mejor con la microperimetría de campo central y patrones de TCO que con la AVMC. La sensibilidad promedio retiniana en las áreas con AF aumentada se encontró disminuida respecto a las áreas con patrón de AF normal. El GPM y el VM no mostraron diferencias significativas entre los diferentes patrones de AF. Se encontró una correlación moderadamente fuerte entre el índice AF/SC basal y el VM en el mes de seguimiento $\left(r_{s=}=0.64\right)$.

Otra diferencia de nuestro estudio respecto al de Vujosevic et al. ${ }^{12}$ estribó en que estos autores clasificaron la AF disminuida como parte del patrón de AF normal, mientras que nosotros la clasificamos como un patrón independiente, estadificándola como AF grado $1 .{ }^{8}$

En otra investigación ${ }^{16}$ se evaluaron los cambios en biomarcadores específicos de imagen (puntos hiperreflécticos intrarretinianos; y AF aumentada) como indicadores potenciales de inflamación retiniana. Se encontró una correlación negativa entre la AF basal (aumentada) y la AVMC en ojos tratados con bevacizumab también en EMD $\left(r_{s}=-0.21\right)$. En nuestro estudio, esta correlación negativa resultó ser aún mayor entre el índice AF/AVMC basal y la AVMC en el segundo mes $\left(r_{s}=-0.78\right)$, así como entre en índice AF/SC basal y la AVMC también en el segundo mes $\left(r_{s}=-0.61\right)$.

Nuestra investigación tiene algunas limitaciones. Los pacientes incluidos tuvieron un seguimiento de solo dos meses. Por otro lado, el uso de sistemas 
de cámara de flash para la obtención de imágenes de AF puede incrementar el riesgo de captación de luz dispersa, produciendo un aumento falso de la AF, fenómeno conocido como pseudoautofluorescencia. Adicionalmente, con las cámaras de flash se obtienen imágenes con bajo contraste, lo que puede conducir a una interpretación errónea de los patrones de AF. ${ }^{6}$ Por último, hay una ausencia de estandarización y base de datos normativa para la AF; por ende, la AF no se considera en la actualidad como un método de exploración de elección para numerosas enfermedades retinianas. Solamente algunos estudio ${ }^{17}$ han revisado sistemáticamente la reproducibilidad y consistencia de las mediciones de la AF.

\section{Conclusiones}

Los índices como el índice AF/AVMC basal o el índice AF/SC basal podrían ser de mayor utilidad como biomarcadores predictivos de la respuesta a este tratamiento que el grado de $\mathrm{AF}$ aislado en pacientes con EMD.

En este sentido, la combinación de dos variables basales en un índice podría aportar un efecto sinérgico importante para la predicción de variables de respuesta anatómica o funcional al tratamiento antiangiogénico en estos pacientes, al menos en el corto plazo.

\section{Agradecimientos}

Los autores desean agradecer a todo el personal técnico de la Clínica David y del Sanatorio La Luz de la ciudad de Morelia, Michoacán, México.

\section{Conflicto de intereses}

Los autores declaran no tener ningún conflicto de intereses.

\section{Financiamiento}

Los autores declaran no tener interés financiero ni autofinanciación del proyecto por parte de la Clínica David, Unidad Oftalmológica en la Ciudad de Morelia, Michoacán.

\section{Responsabilidades éticas}

Protección de personas y animales. Los autores declaran que para esta investigación no realizaron experimentos en seres humanos ni en animales.

Confidencialidad de los datos. Los autores declaran que siguieron los protocolos de su centro de trabajo sobre la publicación de datos de pacientes.

Derecho a la privacidad y consentimiento informado. Los autores declaran que en este artículo no aparecen datos de pacientes.

\section{Bibliografía}

1. Klein R, Knudtson MD, Lee KE, Gangnon R, Klein BE. The Wisconsin Epidemiologic Study of Diabetic Retinopathy XXIII: the twenty-five-year incidence of macular edema in persons with type 1 diabetes. Ophthalmology. 2009:116:497-503.

2. Shaw JE, Sicree RA, Zimmet PZ. Global estimates of the prevalence of diabetes for 2010 and 2030. Diabetes Res Clin Pract. 2010;87:4-14.

3. Klein R, Klein BE, Moss SE, Davis MD, DeMets DL. The Wisconsin epidemiologic study of diabetic retinopathy. IV: diabetic macular edema. Ophthalmology. 1984;91:1464-1474.

4. Midena E, Bini S. Multimodal retinal imaging of diabetic macular edema: toward new paradigms of pathophysiology. Graefes Arch Clin Exp Ophthalmol. 2016;254:1661-1668.

5. Acon D, Wu L. Multimodal imaging in diabetic macular edema. Asia Pac $J$ Ophthalmol (Phila). 2018;7:22-27.

6. Yung M, Klufas MA, Sarraf D. Clinical applications of fundus autofluorescence in retinal disease. Int J Retina Vitreous. 2016;2:12.

7. Frampton GK, Kalita N, Payne L, Colquitt JL, Loveman E, Downes SM, et al. Fundus autofluorescence imaging: systematic review of test accuracy for the diagnosis and monitoring of retinal conditions. Eye (Lond). 2017;31:995-1007.

8. Hernández-Da Mota SE, Melo-Granados EAR, Fromow-Guerra J, Bejar-Cornejo F, Gallego-Pinazo R, Rodríguez-Ayala E. Correlation analysis of fundus autofluorescence, spectral domain optical coherence tomography, and visual function in patients with diabetic macular oedema treated with intravitreal ziv-aflibercept. Eur J Ophthalmol. 2019;29:271-277.

9. Hernández-Da Mota SE, Béjar-Cornejo F, Esquivel-Velázquez M, Lima-Gómez V, González-Saldívar G, Rodríguez-Ayala E, et al. Autofluorescence indexes as biomarkers for antiangiogenic loading dose outcome in diabetic macular edema. Ther Adv Ophthalmol. 2020;12:2515841420942662.

10. Vujosevic S, Casciano M, Pilotto E, Boccassini B, Varano M, Midena E. Diabetic macular edema: fundus autofluorescence and functional correlations. Invest Ophthalmol Vis Sci. 2011;52:442-448.

11. Henández-Da Mota SH, Lima-Gómez V, Rodríguez-Ayala E, Fromow-Guerra JJ, Melo-Granados EAR. Classification of fundus autofluorescence abnormal patterns in diabetic macular edema. Gac Med Mex. 2019:155:458-462.

12. Reznicek L, Dabov S, Haritoglou C, Kampik A, Kernt M, Neubauer AS Green-light fundus autofluorescence in diabetic macular edema. Int $J$ Ophthalmol. 2013;6:75-80.

13. Costa-de Andrade G, de Oliveira-Dias JR, Maia A, Farah ME, Mitne S, Büchele-Rodrigues E. Intravitreal injections of Ziv-aflibercept for diabetic macular edema: a pilot study. Retina. 2016;36:1640-1645.

14. Calvo-Maroto AM, Pérez-Cambrodi RJ, García-Lázaro S, Ferrer-Blasco T, Cerviño A. Ocular autofluorescence in diabetes mellitus. A review. J Diabetes. 2016;8:619-628

15. Shen $Y, X u X$, Liu K. Fundus autofluorescence characteristics in patients with diabetic macular edema. Chin Med J (Engl). 2014;127:1423-1428.

16. Vujosevic S, Torresin T, Bini S, Convento E, Pilotto E, Parrozzani R, et al. Imaging retinal inflammatory biomarkers after intravitreal steroid and anti-VEGF treatment in diabetic macular edema. Acta Ophthalmol. 2017:95:464-471.

17. Chung H, Park B, Shin HJ, Kim HC. Correlation of fundus autofluorescence with spectral-domain optical coherence tomography and vision in diabetic macular edema. Ophthalmology. 2012:119:1056-1065.

18. Pece A, Isola V, Holz F, Milani P, Brancato R. Autofluorescence imaging of cystoid macular edema in diabetic retinopathy. Ophthalmologica. 2010;224:230-235 